\title{
Classification of Abandoned Areas for Solar Energy Projects Using Artificial Intelligence and Quantum Mechanics
}

\author{
David Gabriel de Barros Franco, ${ }^{1,2}$, Maria Teresinha Arns Steiner ${ }^{2}$ \\ ${ }^{1}$ Undergraduate Program in Logistics, Universidade Federal do Tocantins (UFT), Araguaína, Brasil \\ ${ }^{2}$ Graduate Program in Industrial Engineering and Systems, Pontifícia Universidade Católica do Paraná (PUC-PR), Curitiba, Brasil \\ Email: david.franco@uft.edu.br
}

How to cite this paper: Franco, D.G. de B. and Steiner, M.T.A. (2021) Classification of Abandoned Areas for Solar Energy Projects Using Artificial Intelligence and Quantum Mechanics. Journal of Geographic Information System, 13, 318-339.

https://doi.org/10.4236/igis.2021.133018

Received: April 15, 2021

Accepted: May 31, 2021

Published: June 3, 2021

Copyright $\odot 2021$ by author(s) and Scientific Research Publishing Inc. This work is licensed under the Creative Commons Attribution International License (CC BY 4.0).

http://creativecommons.org/licenses/by/4.0/

\begin{abstract}
The increasing demand for energy has intensified recently, requiring alternative sources to fossil fuels, which have become economically and environmentally unfeasible. On the other hand, the increasing land occupation in recent centuries is a growing problem, demanding greater efficiency, particularly in the reuse of abandoned areas, which has become an alternative. An interesting alternative would be installing energy facilities like solar, wind, biomass, and geothermal, in these areas. The objective of this paper is to develop a classification methodology, based on Artificial Intelligence (AI) and Quantum Theory (QT), to automatically carry out the classification of abandoned areas suitable for the settlement of these power plants. Artificial Neural Networks (ANNs) improved by the hybrid algorithm Quantum-behaved Particle Swarm Optimization (QPSO) together with the Levenberg-Marquardt Algorithm (LMA) were used for the classification task. In terms of Mean Squared Error (MSE), the QPSO-LMA approach achieved a decrease of 19.6\% in relation to the classical LMA training with random initial weights. Moreover, the model's accuracy showed an increase of $7.3 \%$ for the QPSO-LMA over the LMA. To validate this new approach, it was also tested on six different datasets available in the UCI Machine Learning Repository and seven classical techniques established in the literature. For the problem of installing photovoltaic plants in abandoned areas, the knowledge acquired with the solar dataset can be extrapolated to other regions.
\end{abstract}

\section{Keywords}

Solar Energy, Soil Reuse, Artificial Neural Networks, Quantum-Behaved Particle Swarm Optimization 


\section{Introduction}

The growing demand for energy has intensified in recent decades [1], requiring alternative sources to fossil fuels [2], which have become economically and environmentally unfeasible [3] [4] [5] [6]. In addition, the increasing occupation of urban and rural space in recent centuries has become a problem [7] [8] [9], requiring greater efficiency in territorial occupation, especially in the reuse of abandoned areas, one of the major current challenges [8] [10]. This problem is more serious when these areas are large and contaminated, constituting a risk to the environment, health and the economy [8] [11] [12]. Therefore, renewable energies, such as solar energy, have proved to be feasible alternatives that enable productivity and social and environmental wellness [13]. They are abundant, clean and, above all, free [14] [15] [16], and can be used in energy generation projects in areas that are currently unused, reconciling the demand for energy and the recovery of these areas.

These abandoned areas, contaminated by substances harmful to the environment and human health, have attracted the attention of governments and non-governmental organizations [17] [18] [19]. Examples can be cited, such as: abandoned mines, generally contaminated by heavy metals [20]; brownfields, which are abandoned industrial or commercial installations [21]; areas of the Superfund, an American federal government program for locating and cleaning up contaminated areas [22]; landfills, mainly for the disposal of food leftovers and packaging [23]; and areas for solid waste, as defined by the Resource Conservation and Recovery Act [24].

Today, around the world, there is installed power of approximately $2180 \mathrm{GW}$. Together, all the 81,533 points analyzed in this work have an estimated potential of more than $6775 \mathrm{GW}$, approximately 3 times what is generated worldwide. This potential is equivalent to over $44,000,000$ tons of carbon dioxide $\left(\mathrm{CO}_{2}\right)$ that would no longer be released into the atmosphere only by the United States (calculated using the Environmental Protection Agency, EPA's, AVoided Emissions and geneRation Tool, AVERT). There are over ten million jobs in the renewable energy sector. With the creation of renewable projects in areas that are currently out of use, it would be possible to multiply this number, making a positive impact on the environment, the economy and society. Currently, renovation projects in these areas are poorly prepared, without the use of automation and data analytics in the decision-making process, which leads to mistaken and often inefficient choices [25].

The objective of this paper is to develop a classification methodology, based on Artificial Intelligence (AI) and Quantum Theory (QT), to automatically carry out the classification of abandoned areas suitable for the settlement of these power plants. The main innovation of this work is the optimization of the initial weights of the ANN using the Quantum-behaved Particle Swarm Optimization (QPSO) metaheuristic together with the Levenberg-Marquardt Algorithm (LMA), called QPSO-LMA algorithm. This innovation will be tested using the 
classification problem of abandoned areas suitable for solar energy facilities as well as another six classic problems from the literature. The results will also be compared with seven classification algorithms established in the literature.

The main contributions of this article are:

- Improvement of Artificial Neural Network (ANN) performance by optimizing initial weights using the Quantum-behaved Particle Swarm Optimization (QPSO);

- Automatic selection of suitable areas for the implementation of renewable energy projects.

This paper is organized as follows. Section 2 presents the theoretical framework of AI and the QPSO algorithm. The methodology for the proposed problem is presented in Section 3. Section 4 shows the results and a discussion about them for the set of solar data; besides, this section shows six datasets from the literature and other seven classical algorithms used for comparison and validation of the proposal. Finally, the conclusions are presented in Section 5.

\section{Theoretical Framework}

Traditional AI aims to represent intelligent behaviors through exact and complete representations of knowledge. However, many real-world problems cannot be described exactly, or the appropriate knowledge of their operation is not available (they are "black boxes"). Computational Intelligence (CI) emerged as a solution to these difficulties, without requiring much a priori knowledge of a problem, producing robust and adaptable (flexible) solutions for diverse scenarios [26].

The field of CI involves paradigms of Computational Science and Operational Research with a view to implementing systems that represent intelligent behavior (which may be defined as the ability to learn and apply this learning to new scenarios) in complex decision-making processes. Of these paradigms, those inspired by nature are predominant, such as ANN, Fuzzy Systems (FS) and Evolutionary Computation (EC), in addition to hybrid systems, which have advantages such as flaw tolerance and incompleteness or inaccuracy of the data used as an input for the algorithms [27].

The strategy that is generally used in CI is the use of approximation techniques that find partial or even incomplete solutions in a feasible space of time and at an acceptable computational cost, because they generally involve high dimensionality problems with many instances [28].

One type of problem addressed by the $\mathrm{CI}$ is the pattern classification problem, such as text recognition [29] [30], image recognition [31], classification of bone fractures [32] [33], endometriosis [34], arrhythmia [35] [36], mineral quality [37] and the identification of medicinal herbs [38], to name a few. Among the many techniques available to address classification problems, we may cite Naïve Bayes [29] [39], Decision Trees [40] [41] [42], Support Vector Machines [42] [43], Gaussian Process Classification [44] [45] [46], k-Nearest Neighbors [47] 
[48] [49] [50], Ensemble methods [51] [52] [53] and Artificial Neural Networks [54] [55] [56] [57] [58].

\subsection{Artificial Neural Networks (ANNs)}

This work will focus on ANNs, as they have advantages such as error tolerance and adaptive learning [59], besides difficulties that could be explored to increase their accuracy [60]. Of these difficulties, we will specifically address the initialization of neural weights, components that store knowledge and are changed during network training [61] [62] [63] [64].

As optimal synaptic weights are difficult to find using analytical methods, it is necessary to use local or global iterative optimization methods [65] to obtain them. Gradient based training algorithms are widely used due to their effectiveness [65]. However, they converge slowly and often cannot escape from local minimums [66].

Historically, synaptic weights were initiated with equal values, which led to their collective convergence, obtaining undesirable outcomes [67]. To break this symmetry, the random initiation method in one defined interval was proposed by Rumelhart, Hinton and Williams (1986) [68], although randomness has been present in ANNs since the Perceptron model, which assumed random connections between neurons [69].

The appropriate initialization of the synaptic weights in the network can reduce the training time and avoid the much undesired local minimums [70] [71] [72] [73], emphasizing that this parameter (synaptic weights) has the greatest effect on the performance of ANNs [74]. Many methods have been developed with a view to overcoming these difficulties, such as those that involve least squares and interval analysis. These methods have been effective in reducing the initial error, although these have proved to be unstable and very often incapable of overcoming local minimums [75]. Therefore, the study of new initialization techniques for ANNs is a very promising field and is the purpose of this article.

One aim of this paper, as already mentioned, is to improve the accuracy of feedforward multilayer perceptron trained with the LMA, which is widely used in ANN training [76] [77], by the optimization of the initial weights using the QPSO metaheuristic (approach called QPSO-LMA). In other hybrid approaches found in the literature, differently from the one used here, metaheuristics are mainly used for tuning ANN parameters or in search of the optimal final weights of the network [78]-[83].

\subsection{Particle Swarm Optimization (PSO)}

PSO is an evolutionary optimization algorithm proposed by Kennedy and Eberhart in the 1990s [84] that uses swarms of particles in its search for the global optimum for a given problem. It was inspired by the social behavior of animals in search of food or prey [28], having as characteristics robustness and efficiency in the search for the global optimum [85]. PSO has been used in many fields of 
knowledge, such as vehicle routing, multi-objective optimization and control systems [86].

The search process performed by the algorithm consists of $N$ particles exploring the neighborhood of the swarm and returning information to their neighbors. It can also be understood as a process that combines searches based on the gradient and on populations, requiring that the function to be optimized should be of the type $f: \mathbb{R}^{D} \rightarrow \mathbb{R}$ [28] [87] [88], where $D$ is the dimension of the problem.

Each particle in the swarm will update its velocity and position according to Equations (1) and (2), where $\omega$ corresponds to the inertia weight, $C_{p}$ and $C_{g}$ are the cognitive learning rate and the social learning rate, respectively, and $\phi_{1}$ and $\phi_{2}$ are uniformly distributed random values in the interval $[0,1]$.

$$
\begin{gathered}
V_{i+1}=\omega \cdot V_{i}+\phi_{1} \cdot C_{p}\left(\text { pbest }_{i}-X_{i}\right)+\phi_{2} \cdot C_{g}\left(\text { gbest }_{i}-X_{i}\right) \\
X_{i+1}=X_{i}+V_{i+1}
\end{gathered}
$$

in Equation (1), pbest and gbest are the memory of the best solution achieved by the particle and by the swarm, respectively.

One of the main disadvantages of the classical version of the PSO algorithm is the selection of its free parameters $\omega, C_{p}$ and $C_{g}$, which leads to longer processing time and still does not guarantee convergence to global minimums [89].

\subsection{Quantum-Behaved Particle Swarm Optimization (QPSO)}

In the quantum version of the PSO algorithm, the state of the particle is given by a wavefunction $\psi(x, t)$, instead of its trajectory (velocity and position). In the quantum realm, the term trajectory is meaningless because of the uncertainty principle [61]. The probability that a particle is in each position can be calculated from its probability density distribution $|\psi(x, t)|^{2}$.

Employing the Monte Carlo method, the particles update its position according to Equation (3) [90]:

$$
\left\{\begin{array}{l}
X_{i+1}=p+\beta \cdot \mid \text { Mbest }_{i}-X_{i} \mid \cdot \ln (1 / u), \text { if } k<0.5 \\
X_{i+1}=p-\beta \cdot \mid \text { Mbest }_{i}-X_{i} \mid \cdot \ln (1 / u), \text { if } k<0.5
\end{array}\right.
$$

where $\beta$ is the contraction-expansion coefficient [91], $u$ and $k$ are random numbers in the range $[0,1]$, generated from a uniform distribution. The global mean best (Mbest) of the population is defined as the mean of the pbest positions of the swarm.

The contraction-expansion coefficient $\beta$ is the only parameter to be tuned in the QPSO algorithm, and this can be done through Bayesian optimization [92]. The local attractor [91] to guarantee convergence of the QPSO algorithm [93] is defined by Equation (4).

$$
p=\left(\varphi_{1} \cdot \text { pbest }+\varphi_{2} \cdot \text { gbest }\right) /\left(\varphi_{1}+\varphi_{2}\right)
$$

where $\varphi_{1}$ and $\varphi_{2}$ are random numbers generated from a uniform distribution in the range $[0,1]$. Alternatively, these numbers can be generated from a positive 
Gaussian distribution with zero mean and unit variance, which leads to a large number of small amplitudes in the movement of the particles [93].

QPSO algorithm is proven to be more effective than other implementations of evolutionary algorithms in most scenarios [90] [94] [95] [96] [97]. In this work, the QPSO algorithm was tested in a more complicated RNA optimization problem and its performance was compared to other methods established in the literature.

\subsection{Hybrid Fuzzy C-Means (HFCM)}

As the classic Fuzzy C-Means (FCM) algorithm assumes the random initialization of the fuzzy partition matrix, a hybrid metaheuristic approach (HFCM) was used with a view to increase the convergence speed of the clustering algorithm. In this work, we used the Differential Evolution metaheuristic for the initialization of the fuzzy partition matrix $\left(\mu_{0}\right)$, since experiments indicated that this metaheuristic can increase up to $23.3 \%$ the training speed of the algorithm [98]. The pseudocode of the HFCM algorithm used for this task is shown in Algorithm 1.

In Algorithm 1, $\mu_{i j} \in[0,1]$ is the fuzzy partition matrix $\left(\sum_{j=1}^{C} \mu_{i j}=1, \forall i\right)$ for the $i$ th instance ( $N$ observations) and the $t$ th centroid ( $C$ centroids, in the interval $[2, N])$, whose $m$ determines the fuzziness of the clustering, usually set in the range [1.25,4], with $m=2$ being the general value [99] [100] [101]. $\left\|x_{i}-c_{j}\right\|^{2}$ is the squared Euclidean distance between the instances $x_{i}$ and the centroids $c_{j}$.

Algorithm 1. HFCM algorithm.

\begin{tabular}{|c|c|}
\hline & Input: Clusters, $m$, Improvement, Max $_{\text {iterations }}$ \\
\hline & Output: $\mu_{\text {best }}$ \\
\hline 1. & $\mu_{0} \leftarrow$ Metaheuristic(Clusters, $m$ ) \\
\hline 2. & while ( $\neg$ StopCondition) \\
\hline 3. & $C_{j}=\sum_{i=1}^{N} \mu_{i j}^{m} X_{i} / \sum_{i=1}^{N} \mu_{i j}^{m}$ \\
\hline 4. & if $\left(\left|\operatorname{Cost}\left(Z_{t}\right)-\operatorname{Cost}\left(Z_{t-1}\right)\right|<\right.$ Improvement $\left.\vee \operatorname{Max}_{\text {ierations }}\right)$ \\
\hline 5. & Stop \\
\hline 6. & Else \\
\hline 7. & $\mu_{i j}=1 / \sum_{k=1}^{C}\left(\frac{\left\|x_{i}-c_{j}\right\|}{\left\|x_{i}-c_{k}\right\|}\right)$ \\
\hline 8. & end \\
\hline 9. & $\mu_{\text {best }} \leftarrow \mu_{i j}$ \\
\hline 10. & end \\
\hline 11. & Return $\left(\mu_{\text {best }}\right)$ \\
\hline
\end{tabular}




\section{Methodology}

In this section, the methodology used in the work is presented, along with the QPSO approach used for ANN initialization (Figure 1).

The methodology was proposed in five main stages: data selection, pre-processing, transformation, data mining and evaluation of the results. In the selection stage, the data were collected from government databases and selected for use in the proposed algorithm. The pre-processing involved filling in missing and removing correlated variables. In the third stage, transformation, the data was normalized to be used as input to the ANN. The data mining stage represents the execution of the algorithms and, finally, the results were analyzed.

\subsection{Data Collection and Pre-Processing}

The solar dataset used in the problem was obtained from the website of the United States Environmental Protection Agency (EPA). The agency oversees the RE-Powering America's Land initiative, which identifies abandoned areas with a potential for recovery and the implementation of renewable energy projects.

With the RE-Powering Mapper tool, it is possible to visualize and download information on renewable energy potential in contaminated lands. Using screening criteria developed in collaboration with the National Renewable Energy Laboratory (NREL) and other state agencies, the EPA has pre-screened over 81,000 sites (at the time of this research) for their renewable energy potential. RE-Powering Mapper features include:

- Screening results for over 81,000 sites for solar, wind, biomass, or geothermal energy;

- Search options by a number of attributes including state, acreage, renewable energy capacity, distance to nearest urban center, and other means;

- Site-specific screening reports;

- Links to the EPA or state program managing the site clean-up.

The raw data totals 81,533 instances, each of which has 13 independent variables, in addition to 3 dependent variables. The independent variables are:

1) Latitude;

2) Longitude;

3) Area, in $m^{2}$;

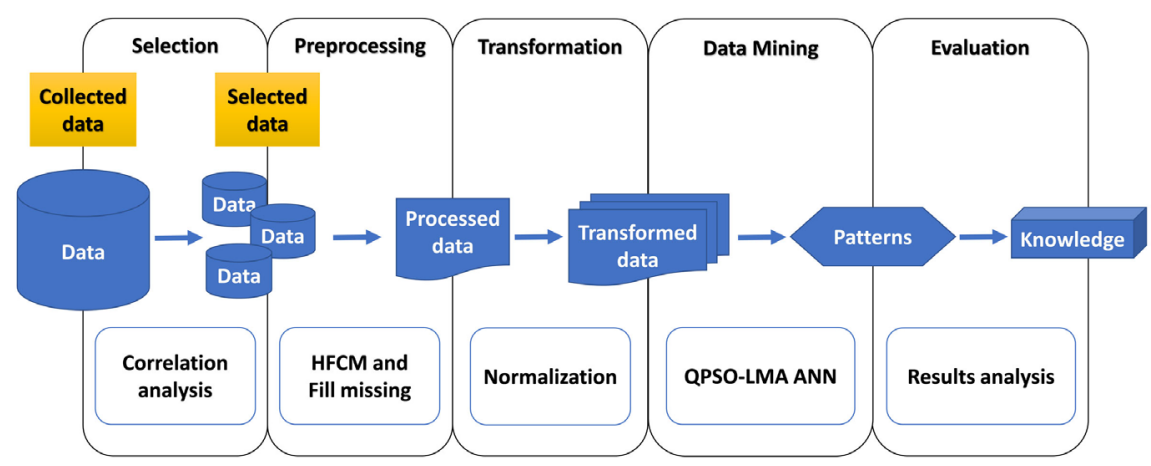

Figure 1. Methodology phases. 
4) Direct Normal Irradiance (DNI), in $\mathrm{kWh} / \mathrm{m}^{2} /$ day;

5) State of the nearest substation (Project or Working);

6) Voltage of the nearest substation, in $\mathrm{kV}$;

7) Distance to the nearest substation, in miles;

8) State of the nearest transmission line (Project or Working);

9) Voltage of the nearest transmission line, in $\mathrm{kV}$;

10) Distance to nearest transmission line, in miles;

11) Distance to nearest road, in miles;

12) Population of nearest urban area;

13) Distance to the nearest urban area, in miles.

The dependent variables have to do with the potential of the location for photovoltaic solar facilities. These areas can be classified into three types:

1) Off-grid: units that do not normally export generated energy to the electricity system and whose solar irradiance is at least $2.5 \mathrm{kWh} / \mathrm{m}^{2} /$ day;

2) Large scale: with at least 300 kilowatts $(\mathrm{kW})$ of power in areas of at least $8000 \mathrm{~m}^{2}$, no farther than $1.6 \mathrm{~km}$ from transmission lines and minimum solar irradiance of $3.5 \mathrm{kWh} / \mathrm{m}^{2} /$ day;

3) Utility scale: operating on a scale of megawatts (MW) in areas larger than $160,000 \mathrm{~m}^{2}$ where the availability of solar irradiance is greater than or equal to 5 $\mathrm{kWh} / \mathrm{m}^{2} /$ day and at up to $16 \mathrm{~km}$ from the transmission lines.

Of the 81,533 points analyzed, one or more of the variables were lacking for 32,429 data points, which needed to be filled. To make up for this deficiency, the average of each variable could be used, which might lead to discrepancies, as the range of the set was significant. With this in mind, it was proposed that the instances could be clustered into smaller sets, using the HFCM algorithm [98], with a view to reducing the scope of each variable in order to perform linear interpolation to supply the missing data.

The new generated data have a lower variance compared to those generated by an interpolation performed for the whole set at once, without the linear interpolation carried out in each cluster. As the Neural Networks demand that all variables have the same dimension in the training and testing phases, it was necessary this stage of data preprocessing.

Compared to the use of a complete data set, without missing data, a deterioration in the results and a consequent loss of accuracy can be assumed. Therefore, this preprocessing phase is important in reducing this deterioration.

It was necessary that no variable, in each instance of any of the clusters, should be left empty. At the same time, the clusters had to be small enough to minimize distortions. Therefore, after many preliminary tests, the number of clusters was experimentally set at 200, with the number of instances per cluster varying from 42 to 1863 , with an average of 407 . The clusters that were formed allowed a reduction in the amplitude of each variable, making the interpolation of the missing data more realistic.

A correlation analysis was conducted of 11 variables (from numbers 3 to 13 , showed at the beginning of this section, because the variables 1 and 2 , 
representing latitude and longitude, were not considered for the classification analysis). A correlation of $91 \%$ between variables number 10 ("Distance to nearest transmission line") and number 11 ("Distance to the nearest road") was found. To avoid the occurrence of multicollinearity, variable number 11 was removed from the classification model, since it is less related to the approached problem.

After collection and preprocessing, the data were separated into input and target sets for the ANN initialized by the QPSO algorithm, using the holdout strategy, considered one of the most reliable when estimating the accuracy of a predictive model [102]. The data were divided into two sets, training and test, with $50 \%$ of the data in each, randomly selected, with a view to a more secure evaluation of the quality of the classification and greater computational simplicity in relation to the k-fold cross-validation [103] [104] [105]. For equivalence, Bayesian Regularization was used in the neural network, which dispenses with the use of the validation set.

\subsection{Proposed Algorithm (QPSO-LMA ANN)}

The proposed initialization process consists of minimizing the mean squared error (MSE) between the target values of the ANN and the values predicted during the learning process, using the QPSO algorithm, called here by QPSO-LMA ANN, or simply, QPSO-LMA. The set of weights and bias, $w$, corresponds to the position of the particles to be optimized by the QPSO algorithm ( $w$ is initialized as an array of random values).

In the pseudocode shown in Algorithm 2, $H$ is the number of neurons in the hidden layer, $N$ is the swarm size for the QPSO algorithm, $D$ is the dimension of the problem (function of the number of variables in the problem: inputs and

Algorithm 2. QPSO pseudo code for ANN initialization.

\begin{tabular}{|c|c|}
\hline & $\begin{array}{l}\text { Input: input,target, } H, \text { iter, } N \\
\text { Output: } w_{\text {best }}, \text { output }\end{array}$ \\
\hline 1. & $i \leftarrow \operatorname{size}($ input $)$ \\
\hline 2. & $t \leftarrow$ size $($ target $)$ \\
\hline 3. & $D \leftarrow(i+1) * H+(H+1) * t$ \\
\hline 4. & $w \leftarrow \operatorname{random}(1, D)$ \\
\hline 5. & $f \leftarrow \operatorname{MSE}(w$, input, target $)$ \\
\hline 6. & while $\neg$ StopCondition \\
\hline 7. & {$[$ output,$w] \leftarrow Q P S O(f, D, N$, iter $)$} \\
\hline 8. & end \\
\hline 9. & $w_{\text {best }} \leftarrow w$ \\
\hline 10. & Return $\left(w_{\text {best }}\right.$, output $)$ \\
\hline
\end{tabular}


targets dimensions, and the number of neurons in the hidden layer), and $f$ is the error function (MSE) that should be minimized.

As output, we will have the values predicted by the network, which will be compared with the target values to measure the percentage accuracy, and the optimized weights $\left(w_{\text {best }}\right)$, which were also used for the initialization of the LMA in a feed-forward ANN, as they have a strong influence on the convergence of the algorithm.

The difference between this methodology and other proposals [106] [107] is in the fact that here the metaheuristic was used in the initialization phase of the algorithm, aiming to bypass the trap of local minimums to which the LMA algorithm is subjected in its initial phase, leading to non-convergence if the search starts far from the global minimum [108]. Thus, the algorithm became more effective in the search for the global minimum without becoming computationally expensive, as in the proposals presented in the literature.

\section{Results and Discussion}

In this section the results of the solar dataset are presented, as well as the other six datasets from the scientific literature. The datasets testing is intended to compare the performance of the proposed technique and its validation alongside what has already been developed regarding classification problems. An Intel i7-2600 $(3.40 \mathrm{GHz})$ computer was used, with $16 \mathrm{~GB}$ of RAM. All the algorithms were implemented in MATLAB, version R2018b.

The number of neurons in the hidden layer and the number of particles in the swarm was determined by Bayesian optimization, which uses Bayesian networks to capture independencies between decision variables of the optimization problem [109].

\subsection{Solar Energy Dataset}

Considering the solar dataset presented in Section 3, the QPSO-LMA hybrid technique achieved a decrease in terms of MSE of $19.6 \%$ in relation to the classical LMA training with random initial weights. An analysis of the percentage accuracy (see Table 1), for the test set, showed an increase of approximately $7.3 \%$ for the QPSO-LMA over the LMA, rising from $75.5 \%$ accurate to $81.0 \%$. Figure 2 shows the classification results and the correctly classified locations for the best result obtained by the QPSO-LMA algorithm.

Figure 2(a) shows the distribution of the three types of abandoned areas throughout the American territory, with a predominance of "Utility" areas in the southwest region. These areas are the largest and have the highest incidence of solar radiation. The southwestern region of the United States is characterized by having large open and desert areas, with arid and sunny climate, leading to a greater concentration of "Utility" type areas in this region. The "Large" type areas are concentrated in the eastern region, being a middle term between the "Utility" and "Off-Grid" areas, taking advantage of medium terrain and with 


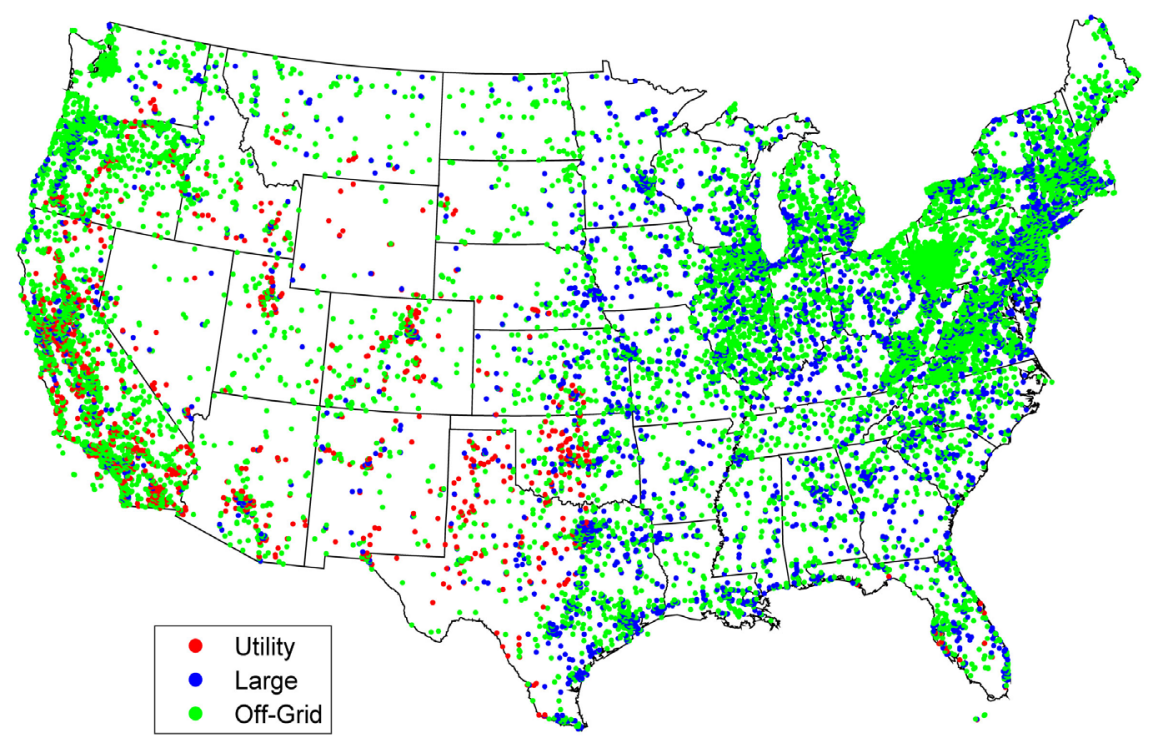

(a)

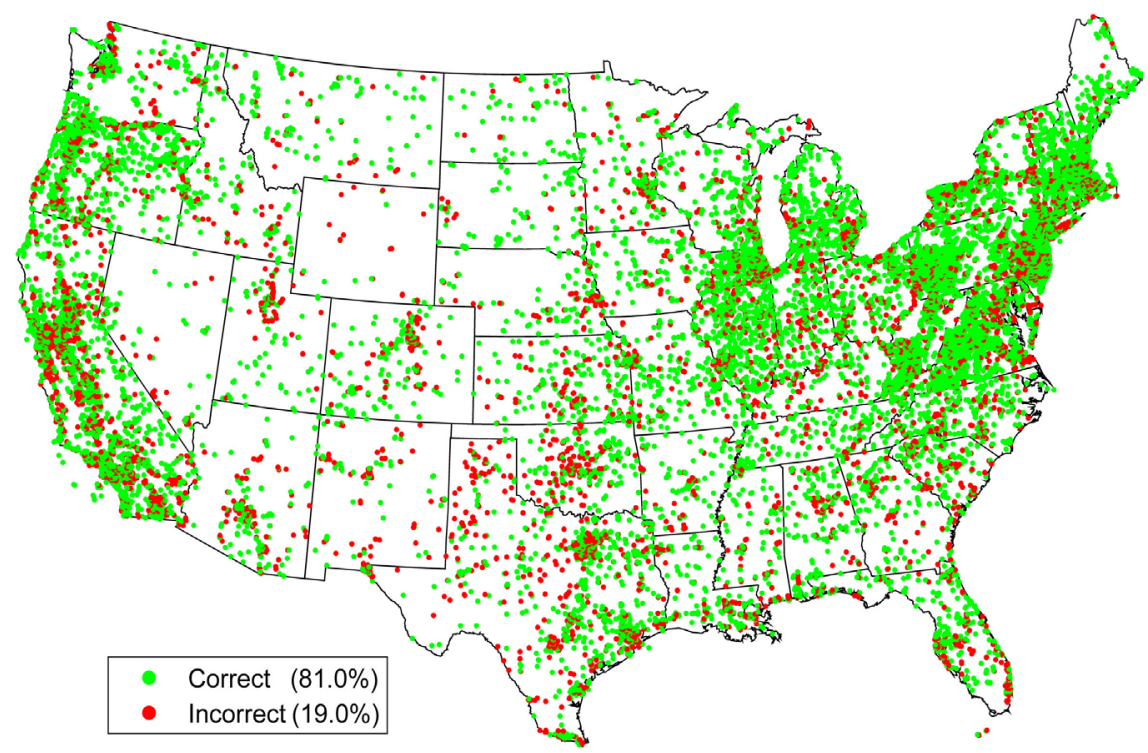

(b)

Figure 2. Classification results (a) and accuracy (b) with QPSO-LMA.

reasonable solar radiation. Finally, the smallest areas, of the "Off-Grid" type, were concentrated in the Northeast and West Coast regions, but also appear throughout the American territory. These small areas seek to make the most of existing resources, including small available land and solar radiation.

Figure 2 (b) shows the correctly classified areas (81.0\%). The incorrectly classified areas (19.0\%) are distributed throughout the American territory, as well as each type of abandoned area, leading to the conclusion that there was no prejudice to the classification due to the imbalance of the dataset. Figure 3 shows that the classification accuracy for off-grid, large scale and utility scale are $83.9 \%$, $71.6 \%$ and $70.9 \%$, respectively. 
Table 1. Results for seven comparative techniques.

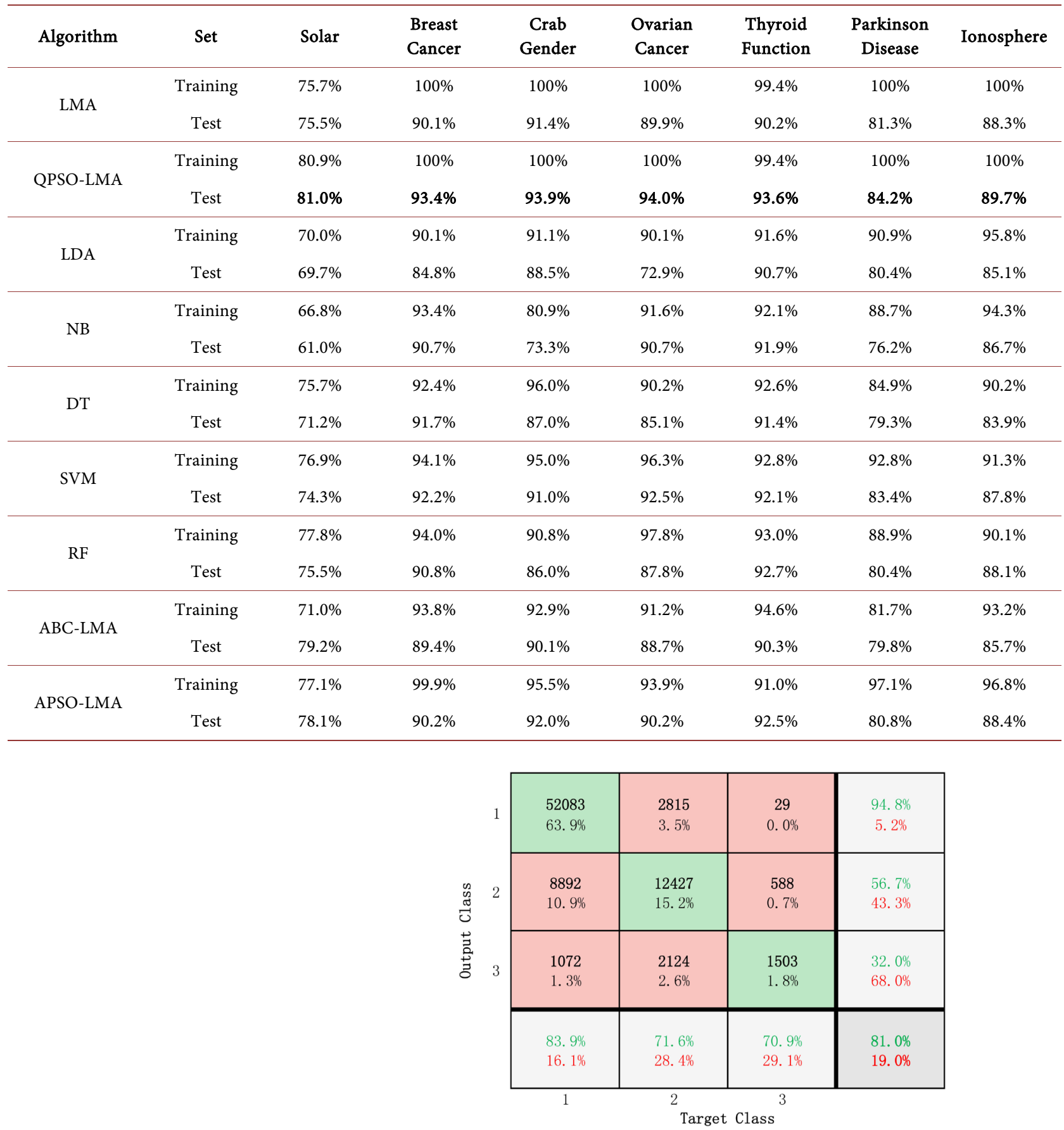

Figure 3. Classification confusion matrix for solar dataset with QPSO-LMA.

As can be seen in Figure 3, errors regarding off-grid areas (class 1) were concentrated in class 2 (large scale) because it has more similar attributes than class 3 (utility scale). The same happened with the other classes.

\subsection{Datasets from Literature}

The proposed algorithm was also tested on six datasets from the literature, the 
most cited in the UCI Machine Learning Repository on the date of data collection, to confirm its effectiveness. The tested datasets, available in the, were:

1) Breast cancer: 9 attributes and 699 instances, classified as benign (65.5\% of cases) or malignant (34.5\% of cases);

2) Crab gender: 6 attributes and 200 instances, classified as male (50\%) or female (50\%);

3) Ovarian cancer: 100 attributes and 216 instances, classified as patients with cancer (56\%) or patients without (44\%);

4) Thyroid function: 21 attributes and 7200 instances, classified as normal (2.3\%), hyperthyroidism (5.1\%) and hypothyroidism (92.6\%);

5) Parkinson's disease: 22 attributes and 195 instances, classified as Parkinson's disease $(75.4 \%)$ or healthy $(24.6 \%)$;

6) Ionosphere: 34 attributes and 351 instances, classified as good radar returns (64.1\%) and bad radar returns (35.9\%).

The proposed algorithm, QPSO-LMA, obtained the best accuracy results for all datasets (see Table 1), including the solar energy dataset. The Thyroid Function dataset, as well as the Solar dataset, is very unbalanced, and even in these databases the proposed algorithm was able to surpass the other algorithms.

The results were also compared using some classical algorithms: Linear Discriminant Analysis (LDA), Naive Bayes (NB), Decision Trees (DT), Support Vector Machines (SVM) and Random Forest (RF), an ensemble learning strategy. These techniques have also been tested with Bayesian parameter optimization. Two hybrid techniques from the literature were also tested, which combine metaheuristics and neural networks with parameter optimization [106] [107]: Artificial Bee Colony Based Levenberg-Marquardt Algorithm (ABC-LMA) and Accelerated Particle Swarm Optimization Based Levenberg-Marquardt Algorithm (APSO-LMA).

All the best results were obtained with the QPSO-LMA algorithm, achieving the objective of the work of proposing a new and efficient initialization strategy of the weights and bias for ANNs in order to solve, with maximum accuracy, the classification of abandoned areas problem which could be suitable for solar energy facilities. Table 1 and Figure 4 show the results.

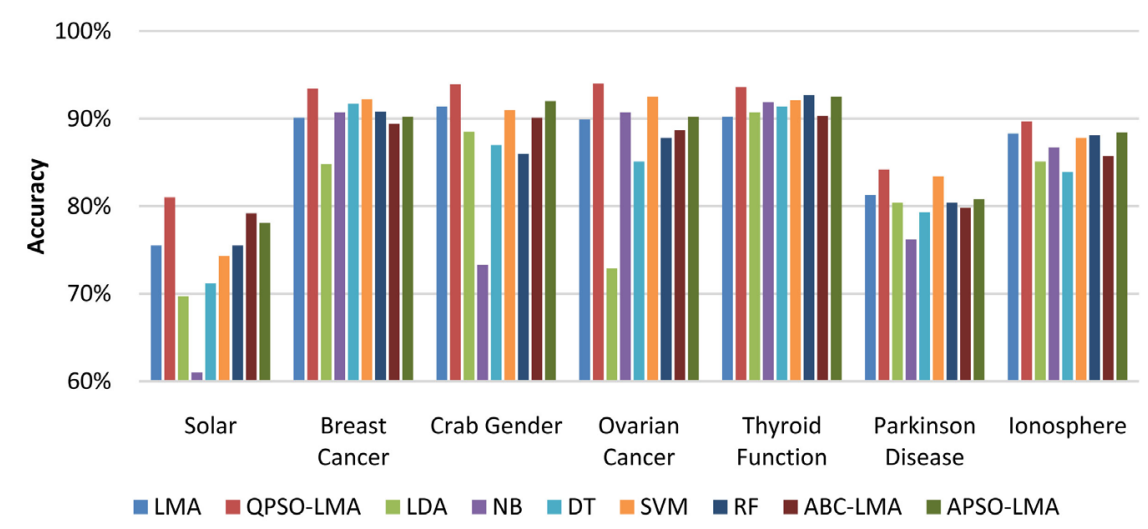

Figure 4. Results for all datasets and algorithms. Source: the authors. 
For the solar energy scenario, the increase in accuracy represents a reduction in error, consequently greater efficiency in choosing the best suitable areas for generating renewable electricity.

It is worth mentioning that, in the references found in the literature, there is normally no division of data in training and testing, with only the training phase, where the error is significantly smaller. Besides that, the QPSO metaheuristic was used only for initializing the weights which reduces the total processing time compared to other hybrid models in the literature.

\section{Conclusions}

The aim of this work was to classify abandoned areas where solar energy facilities could be installed in order to reuse those areas (it is also possible to implement similar decision systems for wind, biomass and geothermal energy, among others). There is enormous energy potential in these abandoned areas, although they are currently neglected. To achieve the classification goal, an ANN was trained with the LMA, in which the initial weights were obtained through the QPSO metaheuristic.

Currently, renovation projects in these areas are poorly prepared, without the use of data analytics in the decision-making process, which leads to inefficient choices. The only criteria used by EPA and NREL to classify abandoned areas are, usually, value ranges with respect to some of the project's variables (estimated capacity, direct normal irradiance, land area and distance to transmission lines).

Using methodologies like the one presented in this work, it is possible to improve this decision process, reducing errors in choosing the most suitable areas, allowing for efficiency gains in the allocation of resources for the implementation of new projects related to solar energy. The areas correctly chosen for renovation will provide greater energy generation and consequently greater return on the investment made, making renewable energies even more competitive.

The results obtained with the solar energy dataset were validated in six of the most cited datasets in the UCI Machine Learning Repository and showed that the proposed strategy was more efficient in all of them. In addition, seven other classification techniques were tested with the seven datasets, with the QPSO-LMA achieving the best result in all cases. This means that QPSO-LMA could improve the accuracy of ANNs, combining the optimization capacity of the QPSO algorithm with the versatility of ANNs in classification problems.

The knowledge acquired by ANNs with the solar dataset can be extrapolated to other regions of the planet, as only technical variables for solar energy were used. This enables the identification of land in locations that do not yet have adequate classification tools. The QPSO-LMA technique could also be used in other classification problems, including other fields of renewable energies, such as wind and geothermal energy and biomass.

Suggestions for future works also include the application of QPSO-LMA algorithm in other databases, since here we had applied just in the seven databases 
(on solar energy, our problem, and on six datasets from the UCI repository) which, of course, is a limitation of this paper. In the same way, it will be interesting to use other Data Mining techniques, to compare to QPSO-LMA proposed algorithm as well as new metaheuristics in addition to QPSO. The application of dataset balancing algorithms, be they undersampling techniques (removal of instances belonging to the over-represented class), or oversampling (generation of new instances, through clustering and interpolation, relative to the under-represented class) could also be used. It will also be possible to test other methods of unrestricted nonlinear optimization as alternatives to the LMA algorithm.

\section{Data Availability Statement}

The data that support the findings of this study are available with the identifier(s) at the private link https://figshare.com/s/754999702alf4919278c.

\section{Acknowledgements}

This study was partially funded by PUC-PR and by the Coordination for the Improvement of Higher Education Personnel-Brazil (CAPES; $1^{\text {st }}$ author) and by the National Council for Scientific and Technological Development-Brazil (CNPq; $2^{\text {nd }}$ author).

\section{Conflicts of Interest}

The authors declare no conflicts of interest regarding the publication of this paper.

\section{References}

[1] U.S. Energy Information Administration (2017) International Energy Outlook 2017.

[2] Griffin, P.W., Hammond, G.P. and Norman, J.B. (2018) Industrial Energy Use and Carbon Emissions Reduction in the Chemicals Sector: A UK Perspective. Applied Energy, 227, 587-602. https://doi.org/10.1016/j.apenergy.2017.08.010

[3] Almeida, C.M.V.B., Agostinho, F., Huisingh, D. and Giannetti, B.F. (2017) Cleaner Production towards a Sustainable Transition. Journal of Cleaner Production, 142, 1-7. https://doi.org/10.1016/j.jclepro.2016.10.094

[4] Cadez, S. and Czerny, A. (2016) Climate Change Mitigation Strategies in Carbon-Intensive Firms. Journal of Cleaner Production, 112, 4132-4143. https://doi.org/10.1016/j.jclepro.2015.07.099

[5] Perea-Moreno, A.-J., García-Cruz, A., Novas, N. and Manzano-Agugliaro, F. (2017) Rooftop Analysis for Solar Flat Plate Collector Assessment to Achieving Sustainability Energy. Journal of Cleaner Production, 148, 545-554. https://doi.org/10.1016/j.jclepro.2017.02.019

[6] Manan, Z.A., Mohd Nawi, W.N.R., Wan Alwi, S.R. and Klemeš, J.J. (2017) Advances in Process Integration Research for $\mathrm{CO}_{2}$ Emission Reduction-A Review. Journal of Cleaner Production, 167, 1-13. https://doi.org/10.1016/j.jclepro.2017.08.138

[7] Lambin, E.F. and Meyfroidt, P. (2011) Global Land Use Change, Economic Globa- 
lization, and the Looming Land Scarcity. The Proceedings of the National Academy of Sciences of the United States of America, 108, 3465-3472. https://doi.org/10.1073/pnas.1100480108

[8] Morio, M., Schädler, S. and Finkel, M. (2013) Applying a Multi-Criteria Genetic Algorithm Framework for Brownfield Reuse Optimization: Improving Redevelopment Options Based on Stakeholder Preferences. Journal of Environmental Management, 130, 331-346. https://doi.org/10.1016/j.jenvman.2013.09.002

[9] Goldewijk, K.K., Beusen, A., Doelman, J. and Stehfest, E. (2017) Anthropogenic Land Use Estimates for the Holocene-HYDE 3.2. Earth System Science Data, 9, 927-953. https://doi.org/10.5194/essd-9-927-2017

[10] Nuissl, H. and Schroeter-Schlaack, C. (2009) On the Economic Approach to the Containment of Land Consumption. Environmental Science and Policy, 12, 270-280. https://doi.org/10.1016/j.envsci.2009.01.008

[11] Apostolidis, N. and Hutton, N. (2006) Integrated Water Management in Brownfield Sites-More Opportunities than You Think. Desalination, 188, 169-175.

https://doi.org/10.1016/j.desal.2005.04.114

[12] Cao, K. and Guan, H. (2007) Brownfield Redevelopment toward Sustainable Urban Land Use in China. Chinese Geographical Science, 17, 127-134. https://doi.org/10.1007/s11769-007-0127-5

[13] Zappa, W., Junginger, M. and van den Broek, M. (2019) Is a 100\% Renewable European Power System Feasible by 2050? Applied Energy, 233-234, 1027-1050. https://doi.org/10.1016/j.apenergy.2018.08.109

[14] González, M.O.A., Gonçalves, J.S. and Vasconcelos, R.M. (2017) Sustainable Development: Case Study in the Implementation of Renewable Energy in Brazil. Journal of Cleaner Production, 142, 461-475. https://doi.org/10.1016/j.jclepro.2016.10.052

[15] Lima, F., Ferreira, P. and Vieira, F. (2013) Strategic Impact Management of Wind Power Projects. Renewable and Sustainable Energy Reviews, 25, 277-290.

https://doi.org/10.1016/j.rser.2013.04.010

[16] Fernández-García, A., Rojas, E., Pérez, M., Silva, R., Hernández-Escobedo, Q. and Manzano-Agugliaro, F. (2015) A Parabolic-Trough Collector for Cleaner Industrial Process Heat. Journal of Cleaner Production, 89, 272-285. https://doi.org/10.1016/j.jclepro.2014.11.018

[17] Bergius, K. and Öberg, T. (2007) Initial Screening of Contaminated Land: A Comparison of US and Swedish Methods. Environmental Management, 39, 226-234. https://doi.org/10.1007/s00267-006-0005-4

[18] Zhu, J., Liao, S., Lei, Z. and Li, S.Z. (2017) Multi-Label Convolutional Neural Network Based Pedestrian Attribute Classification. Image and Vision Computing, 58, 224-229. https://doi.org/10.1016/j.imavis.2016.07.004

[19] Hartmann, B., Török, S., Börcsök, E. and Oláhné Groma, V. (2014) Multi-Objective Method for Energy Purpose Redevelopment of Brownfield Sites. Journal of Cleaner Production, 82, 202-212. https://doi.org/10.1016/j.jclepro.2014.07.002

[20] Kovacs, H. and Szemmelveisz, K. (2017) Disposal Options for Polluted Plants Grown on Heavy Metal Contaminated Brownfield Lands-A Review. Chemosphere, 166, 8-20. https://doi.org/10.1016/j.chemosphere.2016.09.076

[21] U.S. Government Publishing Office (2002) Public Law 107-118-Small Business Liability Relief and Brownfields Revitalization Act. HR 2869, 1-27. https://goo.gl/UK19n2

[22] U.S. Government Publishing Office (2015) 42 U.S.C. 9601-9628-Hazardous 
Substances Releases, Liability, Compensation. United States Code, 2012 Ed Suppl 3, Title 42-Public Heal Welfare, Subchapter I, 6945-7034.

https://goo.gl/y0ki6N

[23] Rong, L., Zhang, C., Jin, D. and Dai, Z. (2017) Assessment of the Potential Utilization of Municipal Solid Waste from a Closed Irregular Landfill. Journal of Cleaner Production, 142, 413-419. https://doi.org/10.1016/j.jclepro.2015.10.050

[24] U.S. Government Publishing Office. (2011) 40 C.F.R. 239-282-Solid Wastes. Code Fed Regul (Annual Ed). https://goo.gl/UBCLDF

[25] Justin, M.Z. and Zupančič, M. (2009) Combined Purification and Reuse of Landfill Leachate by Constructed Wetland and Irrigation of Grass and Willows. Desalination, 246, 157-168. https://doi.org/10.1016/j.desal.2008.03.049

[26] Siddique, N. and Adeli, H. (2013) Computational Intelligence: Synergies of Fuzzy Logic, Neural Networks and Evolutionary Computing. John Wiley \& Sons, Ltd., Oxford. https://doi.org/10.1002/9781118534823

[27] Kacprzyk, J. and Pedrycz, W., Eds. (2015) Springer Handbook of Computational Intelligence. Springer, Berlin. https://doi.org/10.1007/978-3-662-43505-2

[28] Kruse, R., Borgelt, C., Braune, C., Mostaghim, S. and Steinbrecher, M. (2016) Computational Intelligence: A Methodological Introduction. 2nd Edition, Springer, London. https://doi.org/10.1007/978-1-4471-7296-3

[29] Hadi, W., Al-Radaideh, Q.A. and Alhawari, S. (2018) Integrating Associative Rule-Based Classification with Naïve Bayes for Text Classification. Applied Soft Computing, 69, 344-356. https://doi.org/10.1016/j.asoc.2018.04.056

[30] Mirończuk, M.M. and Protasiewicz, J. (2018) A Recent Overview of the State-of-the-Art Elements of Text Classification. Expert Systems with Applications, 106, 36-54. https://doi.org/10.1016/j.eswa.2018.03.058

[31] Yuan, H., Li, J., Lai, L.L. and Tang, Y.Y. (2018) Graph-Based Multiple Rank Regression for Image Classification. Neurocomputing, 315, 394-404. https://doi.org/10.1016/j.neucom.2018.07.032

[32] Agrawal, A. (2018) Unified Classification of Open Fractures: Based on Gustilo and OTA Classification Schemes. Injury, 49, 1526-1531. https://doi.org/10.1016/j.injury.2018.06.007

[33] Soni, A., Gupta, R., Gupta, S., Kansay, R. and Kapoor, L. (2018) Mechanism of Injury Based Classification of Proximal Tibia Fractures. Journal of Clinical Orthopaedics and Trauma, 10, 785-788. https://doi.org/10.1016/j.jcot.2018.08.012

[34] Andres, M.P., Borrelli, G.M. and Abrão, M.S. (2018) Endometriosis Classification according to Pain Symptoms: Can the ASRM Classification Be Improved? Best Practice and Research Clinical Obstetrics and Gynaecology, 51, 111-118. https://doi.org/10.1016/j.bpobgyn.2018.06.003

[35] Cappe, M., Deruelle, P., Depret, S., Houfflin-Debarge, V., Ghesquière, L. and Garabedian, C. (2018) Fetal Heart Rate Classification in Routine Use: Do Your Prefer a 3-Tier or a 5-Tier Classification? Journal of Gynecology Obstetrics and Human Reproduction, 47, 477-480. https://doi.org/10.1016/j.jogoh.2018.08.001

[36] Sannino, G. and De Pietro, G. (2018) A Deep Learning Approach for ECG-Based Heartbeat Classification for Arrhythmia Detection. Future Generation Computer Systems, 86, 446-455. https://doi.org/10.1016/j.future.2018.03.057

[37] Tutmez, B. (2018) Bauxite Quality Classification by Shrinkage Methods. Journal of Geochemical Exploration, 191, 22-27. https://doi.org/10.1016/i.gexplo.2018.05.002

[38] Ren, L., Guo, M. and Pang, X. (2018) Identification and Classification of Medicinal 
Plants in Epimedium. Chinese Herbal Medicines, 10, 249-254. https://doi.org/10.1016/j.chmed.2018.05.004

[39] Kim, H., Kim, J., Kim, J. and Lim, P. (2018) Towards Perfect Text Classification with Wikipedia-Based Semantic Naïve Bayes Learning. Neurocomputing, 315, 128-134. https://doi.org/10.1016/j.neucom.2018.07.002

[40] Wang, C. and Wang, J.Z. (2018) Cloud-Service Decision Tree Classification for Education Platform. Cognitive Systems Research, 52, 234-239. https://doi.org/10.1016/j.cogsys.2018.06.021

[41] Beucher, A., Møller, A.B. and Greve, M.H. (2019) Artificial Neural Networks and Decision Tree Classification for Predicting Soil Drainage Classes in Denmark. Geoderma, 352, 351-359.

[42] Wu, W., Li, A.-D., He, X.-H., Ma, R., Liu, H.-B. and Lv, J.-K. (2018) A Comparison of Support Vector Machines, Artificial Neural Network and Classification Tree for Identifying Soil Texture Classes in Southwest China. Computers and Electronics in Agriculture, 144, 86-93. https://doi.org/10.1016/j.compag.2017.11.037

[43] Mancuhan, K. and Clifton, C. (2018) Support Vector Classification with $\ell$-Diversity. Computers and Security, 77, 653-665. https://doi.org/10.1016/j.cose.2017.12.010

[44] Rinta-Koski, O.-P., Särkkä, S., Hollmén, J., Leskinen, M. and Andersson, S. (2018) Gaussian Process Classification for Prediction of In-Hospital Mortality among Preterm Infants. Neurocomputing, 298, 134-141.

https://doi.org/10.1016/j.neucom.2017.12.064

[45] Andrade, D., Tamura, A. and Tsuchida, M. (2018) Exploiting Covariate Embeddings for Classification Using Gaussian Processes. Pattern Recognition Letters, 104, 8-14. https://doi.org/10.1016/j.patrec.2018.01.011

[46] Sovizi, J., Mathieu, K.B., Thrower, S.L., Stefan, W., Hazle, J.D. and Fuentes, D. (2017) Gaussian Process Classification of Superparamagnetic Relaxometry Data: Phantom Study. Artificial Intelligence in Medicine, 82, 47-59.

https://doi.org/10.1016/j.artmed.2017.07.001

[47] Müller, P., Salminen, K., Nieminen, V., Kontunen, A., Karjalainen, M., Isokoski, P., et al. (2019) Scent Classification by K Nearest Neighbors Using Ion-Mobility Spectrometry Measurements. Expert Systems with Applications, 115, 593-606. https://doi.org/10.1016/j.eswa.2018.08.042

[48] Zhang, X., Li, Y., Kotagiri, R., Wu, L., Tari, Z. and Cheriet, M. (2017) KRNN: K Rare-Class Nearest Neighbour Classification. Pattern Recognition, 62, 33-44. https://doi.org/10.1016/j.patcog.2016.08.023

[49] Guo, Y., Han, S., Li, Y., Zhang, C. and Bai, Y. (2018) K-Nearest Neighbor Combined with Guided Filter for Hyperspectral Image Classification. Procedia Computer Science, 129, 159-165. https://doi.org/10.1016/j.procs.2018.03.066

[50] Gallego, A.-J., Calvo-Zaragoza, J., Valero-Mas, J.J. and Rico-Juan, J.R. (2018) Clustering-Based $K$-Nearest Neighbor Classification for Large-Scale Data with Neural Codes Representation. Pattern Recognition, 74, 531-543.

https://doi.org/10.1016/j.patcog.2017.09.038

[51] Feng, X., Xiao, Z., Zhong, B., Qiu, J. and Dong, Y. (2018) Dynamic Ensemble Classification for Credit Scoring Using Soft Probability. Applied Soft Computing, 65, 139-151. https://doi.org/10.1016/j.asoc.2018.01.021

[52] Jackowski, K. (2018) New Diversity Measure for Data Stream Classification Ensembles. Engineering Applications of Artificial Intelligence, 74, 23-34. https://doi.org/10.1016/j.engappai.2018.05.006 
[53] Ankit and Saleena, N. (2018) An Ensemble Classification System for Twitter Sentiment Analysis. Procedia Computer Science, 132, 937-946. https://doi.org/10.1016/j.procs.2018.05.109

[54] Simoncini, M., Taccari, L., Sambo, F., Bravi, L., Salti, S. and Lori, A. (2018) Vehicle Classification from Low-Frequency GPS Data with Recurrent Neural Networks. Transportation Research Part C: Emerging Technologies, 91, 176-191. https://doi.org/10.1016/j.trc.2018.03.024

[55] Sharma, N., Jain, V. and Mishra, A. (2018) An Analysis of Convolutional Neural Networks for Image Classification. Procedia Computer Science, 132, 377-384. https://doi.org/10.1016/j.procs.2018.05.198

[56] Li, H., Li, G., Ji, X. and Shi, L. (2018) Deep Representation via Convolutional Neural Network for Classification of Spatiotemporal Event Streams. Neurocomputing, 299, 1-9. https://doi.org/10.1016/j.neucom.2018.02.019

[57] de Mesquita Sá, J.J.J., , Backes, A.R. and Bruno, O.M. (2018) Randomized Neural Network Based Descriptors for Shape Classification. Neurocomputing, 312, 201-209. https://doi.org/10.1016/j.neucom.2018.05.099

[58] Gotsopoulos, A., Saarimäki, H., Glerean, E., Jääskeläinen, I.P., Sams, M., Nummenmaa, L., et al. (2018) Reproducibility of Importance Extraction Methods in Neural Network Based fMRI Classification. Neuroimage, 181, 44-54. https://doi.org/10.1016/j.neuroimage.2018.06.076

[59] Marugán, A.P., Márquez, F.P.G., Perez, J.M.P. and Ruiz-Hernández, D. (2018) A Survey of Artificial Neural Network in Wind Energy Systems. Applied Energy, 228, 1822-1836. https://doi.org/10.1016/j.apenergy.2018.07.084

[60] Cao, W., Wang, X., Ming, Z. and Gao, J. (2018) A Review on Neural Networks with Random Weights. Neurocomputing, 275, 278-287. https://doi.org/10.1016/j.neucom.2017.08.040

[61] Ojha, V.K., Abraham, A. and Snášel, V. (2017) Metaheuristic Design of Feedforward Neural Networks: A Review of Two Decades of Research. Engineering Applications of Artificial Intelligence, 60, 97-116. https://doi.org/10.1016/j.engappai.2017.01.013

[62] Prieto, A., Prieto, B., Ortigosa, E.M., Ros, E., Pelayo, F., Ortega, J., et al. (2016) Neural Networks: An Overview of Early Research, Current Frameworks and New Challenges. Neurocomputing, 214, 242-268. https://doi.org/10.1016/j.neucom.2016.06.014

[63] Basheer, I. and Hajmeer, M. (2000) Artificial Neural Networks: Fundamentals, Computing, Design, and Application. Journal of Microbiological Methods, 43, 3-31. https://doi.org/10.1016/S0167-7012(00)00201-3

[64] Almási, A.-D., Woźniak, S., Cristea, V., Leblebici, Y. and Engbersen, T. (2016) Review of Advances in Neural Networks: Neural Design Technology Stack. Neurocomputing, 174, 31-41. https://doi.org/10.1016/j.neucom.2015.02.092

[65] Erdogmus, D., Fontenla-Romero, O., Principe, J.C., Alonso-Betanzos, A., Castillo, E. (2005) Linear-Least-Squares Initialization of Multilayer Perceptrons through Backpropagation of the Desired Response. IEEE Transactions on Neural Networks, 16, 325-337. https://doi.org/10.1109/TNN.2004.841777

[66] Nasr, M.B. and Chtourou, M. (2011) A Self-Organizing Map-Based Initialization for Hybrid Training of Feedforward Neural Networks. Applied Soft Computing, 11, 4458-4464. https://doi.org/10.1016/j.asoc.2011.05.017

[67] Sodhi, S.S. and Chandra, P. (2014) Interval Based Weight Initialization Method for Sigmoidal Feedforward Artificial Neural Networks. AASRI Procedia, 6, 19-25. 
https://doi.org/10.1016/j.aasri.2014.05.004

[68] Rumelhart, D.E., Hinton, G.E. and Williams, R.J. (1986) Learning Representations by Back-Propagating Errors. Nature, 323, 533-536.

https://doi.org/10.1038/323533a0

[69] Rosenblatt, F. (1958) The Perceptron: A Probabilistic Model for Information Storage and Organization in the Brain. Psychological Review, 65, 386-408. https://doi.org/10.1037/h0042519

[70] Adam, S.P., Karras, D.A., Magoulas, G.D. and Vrahatis, M.N. (2014) Solving the Linear Interval Tolerance Problem for Weight Initialization of Neural Networks. Neural Networks, 54, 17-37. https://doi.org/10.1016/j.neunet.2014.02.006

[71] Talaśka, T., Kolasa, M., Długosz, R. and Farine, P.-A. (2015) An Efficient Initialization Mechanism of Neurons for Winner Takes all Neural Network Implemented in the CMOS Technology. Applied Mathematics and Computation, 267, 119-138. https://doi.org/10.1016/j.amc.2015.04.123

[72] Qing, S. (2011) Robust Initialization of a Jordan Network with Recurrent Constrained Learning. IEEE Transactions on Neural Networks, 22, 2460-2473. https://doi.org/10.1109/TNN.2011.2168423

[73] Napolitano, G., Serinaldi, F. and See, L. (2011) Impact of EMD Decomposition and Random Initialisation of Weights in ANN Hindcasting of Daily Stream Flow Series: An Empirical Examination. Journal of Hydrology, 406, 199-214. https://doi.org/10.1016/j.jhydrol.2011.06.015

[74] Dolezel, P., Skrabanek, P. and Gago, L. (2016) Weight Initialization Possibilities for Feedforward Neural Network with Linear Saturated Activation Functions. IFAC-PapersOnLine, 49, 49-54. https://doi.org/10.1016/j.ifacol.2016.12.009

[75] Qiao, J., Li, S. and Li, W. (2016) Mutual Information Based Weight Initialization Method for Sigmoidal Feedforward Neural Networks. Neurocomputing, 207, 676-683. https://doi.org/10.1016/j.neucom.2016.05.054

[76] Daniel, L., Chaturvedi, K.T. and Kolhe, M.L. (2018) Dynamic Economic Load Dispatch Using Levenberg Marquardt Algorithm. Energy Procedia, 144, 95-103. https://doi.org/10.1016/j.egypro.2018.06.013

[77] Mammadli, S. (2017) Financial Time Series Prediction Using Artificial Neural Network Based on Levenberg-Marquardt Algorithm. Procedia Computer Science, 120, 602-607. https://doi.org/10.1016/j.procs.2017.11.285

[78] Hraiba, A., Touil, A. and Mousrij, A. (2020) Artificial Neural Network Based Hybrid Metaheuristics for Reliability Analysis. IFAC-PapersOnLine, 53, 654-660. https://doi.org/10.1016/j.ifacol.2020.06.109

[79] Wan, P., Zou, H., Wang, K. and Zhao, Z. (2020) Research on Hot Deformation Behavior of Zr-4 Alloy Based on PSO-BP Artificial Neural Network. Journal of Alloys and Compounds, 826, Article ID: 154047. https://doi.org/10.1016/j.jallcom.2020.154047

[80] Deng, Y., Xiao, H., Xu, J. and Wang, H. (2019) Prediction Model of PSO-BP Neural Network on Coliform Amount in Special Food. Saudi Journal of Biological Sciences, 26, 1154-1160. https://doi.org/10.1016/j.sjbs.2019.06.016

[81] Panda, N. and Majhi, S.K. (2020) Improved Spotted Hyena Optimizer with Space Transformational Search for Training Pi-Sigma Higher Order Neural Network. Computational Intelligence, 36, 320-350. https://doi.org/10.1111/coin.12272

[82] Abedinia, O., Amjady, N. and Ghadimi, N. (2018) Solar Energy Forecasting Based on Hybrid Neural Network and Improved Metaheuristic Algorithm. Computational 
Intelligence, 34, 241-260. https://doi.org/10.1111/coin.12145

[83] Gong, S., Gao, W. and Abza, F. (2020) Brain Tumor Diagnosis Based on Artificial Neural Network and a Chaos Whale Optimization Algorithm. Computational Intelligence, 36, 259-275. https://doi.org/10.1111/coin.12259

[84] Kennedy, J. and Eberhart, R. (1995) Particle Swarm Optimization. Proceedings of ICNN95-International Conference on Neural Networks, Vol. 4, IEEE, Perth, WA, 27 November-1 December 1995, 1942-1948. https://doi.org/10.1109/ICNN.1995.488968

[85] Zhang, H., Yuan, M., Liang, Y. and Liao, Q. (2018) A Novel Particle Swarm Optimization Based on Prey-Predator Relationship. Applied Soft Computing, 68, 202-218. https://doi.org/10.1016/j.asoc.2018.04.008

[86] Chen, Y., Li, L., Peng, H., Xiao, J. and Wu, Q. (2018) Dynamic Multi-Swarm Differential Learning Particle Swarm Optimizer. Swarm and Evolutionary Computation, 39, 209-221. https://doi.org/10.1016/j.swevo.2017.10.004

[87] Engelbrecht, A.P. (2007) Computational Intelligence: An Introduction. 2nd Edition, John Wiley \& Sons, Ltd., Hoboken. https://doi.org/10.1002/9780470512517

[88] Brownlee, J. (2011) Clever Algorithms. LuLu.com, Raleigh, NC.

[89] Zhu, X. and Xu, B. (2012) Urban Water Consumption Forecast Based on QPSO-RBF Neural Network. 2012 Eighth International Conference on Computational Intelligence and Security, Guangzhou, 17-18 November 2012, 233-236. https://doi.org/10.1109/CIS.2012.59

[90] Sun, J., Feng, B. and Xu, W. (2004) Particle Swarm Optimization with Particles Having Quantum Behavior. Proceedings of the 2004 Congress on Evolutionary Computation, Vol. 1, IEEE, Portland, 19-23 June 2004, 325-331. https://doi.org/10.1109/CEC.2004.1330875

[91] Clerc, M. and Kennedy, J. (2002) The Particle Swarm-Explosion, Stability, and Convergence in a Multidimensional Complex Space. IEEE Transactions on Evolutionary Computation, 6, 58-73. https://doi.org/10.1109/4235.985692

[92] Kouziokas, G.N. (2020) A New W-SVM Kernel Combining PSO-Neural Network Transformed Vector and Bayesian Optimized SVM in GDP Forecasting. Engineering Applications of Artificial Intelligence, 92, Article ID: 103650. https://doi.org/10.1016/j.engappai.2020.103650

[93] Coelho, L.d.S. (2010) Gaussian Quantum-Behaved Particle Swarm Optimization Approaches for Constrained Engineering Design Problems. Expert Systems with Applications, 37, 1676-1683. https://doi.org/10.1016/j.eswa.2009.06.044

[94] Sun, J., Xu, W. and Feng, B. (2005) Adaptive Parameter Control for Quantum-Behaved Particle Swarm Optimization on Individual Level. 2005 IEEE International Conference on Systems, Man and Cybernetics, Vol. 4, Waikoloa, 12 October 2005, 3049-3054. https://doi.org/10.1109/ICSMC.2005.1571614

[95] Sun, J., Xu, W. and Feng, B. (2004) A Global Search Strategy of Quantum-Behaved Particle Swarm Optimization. IEEE Conference on Cybernetics and Intelligence Systems 2004, Vol. 1, Singapore, 1-3 December 2004, 111-116. https://doi.org/10.1109/ICCIS.2004.1460396

[96] Xi, M., Sun, J. and Xu, W. (2008) An Improved Quantum-Behaved Particle Swarm Optimization Algorithm with Weighted Mean Best Position. Applied Mathematics and Computation, 205, 751-759. https://doi.org/10.1016/j.amc.2008.05.135

[97] Fang, W., Sun, J., Ding, Y., Wu, X. and Xu, W. (2010) A Review of Quantum-Behaved Particle Swarm Optimization. IETE Technical Review, 27, 336-348. 
https://doi.org/10.4103/0256-4602.64601

[98] Franco D.G.d.B. and Steiner M.T.A. (2018) Clustering of Solar Energy Facilities Using a Hybrid Fuzzy C-Means Algorithm Initialized by Metaheuristics. Journal of Cleaner Production, 191, 445-457. https://doi.org/10.1016/j.jclepro.2018.04.207

[99] Zhou, K., Yang, S. and Shao, Z. (2017) Household Monthly Electricity Consumption Pattern Mining: A Fuzzy Clustering-Based Model and a Case Study. Journal of Cleaner Production, 141, 900-908. https://doi.org/10.1016/j.jclepro.2016.09.165

[100] Wu, K.-L. (2012) Analysis of Parameter Selections for Fuzzy C-Means. Pattern Recognition, 45, 407-415. https://doi.org/10.1016/j.patcog.2011.07.012

[101] Zhou, K., Fu, C. and Yang, S. (2014) Fuzziness Parameter Selection in Fuzzy C-Means: The Perspective of Cluster Validation. Science China Information Sciences, 57, 1-8. https://doi.org/10.1007/s11432-014-5146-0

[102] Lee, L.C., Liong, C.-Y. and Jemain, A.A. (2018) Validity of the Best Practice in Splitting Data for Hold-Out Validation Strategy as Performed on the Ink Strokes in the Context of Forensic Science. Microchemical Journal, 139, 125-133.

https://doi.org/10.1016/j.microc.2018.02.009

[103] Yadav, S. and Shukla, S. (2016) Analysis of K-Fold Cross-Validation over Hold-Out Validation on Colossal Datasets for Quality Classification. 2016 IEEE 6th International Conference on Advanced Computing, Bhimavaram, 27-28 February 2016, 78-83. https://doi.org/10.1109/IACC.2016.25

[104] Arlot, S. and Celisse, A. (2010) A Survey of Cross-Validation Procedures for Model Selection. Statistics Surveys, 4, 40-79. https://doi.org/10.1214/09-SS054

[105] Massart, P. (2007) Concentration Inequalities and Model Selection. Vol. 1896, Springer, Berlin.

[106] Nawi, N.M., Khan, A., Rehman, M.Z., Aziz, M.A., Herawan, T. and Abawajy, J.H. (2014) An Accelerated Particle Swarm Optimization Based Levenberg Marquardt Back Propagation Algorithm. In: Loo, C.K., Yap, K.S., Wong, K.W., Teoh, A. and Huang, K., Eds., Neural Information Processing, International Conference on Neural Information Processing 2014, Vol. 8835, Springer, Cham, 245-253. https://doi.org/10.1007/978-3-319-12640-1 30

[107] Dilmen, E., Yilmaz, S. and Beyhan, S. (2017) Chapter 5. An Intelligent Hybridization of $\mathrm{ABC}$ and LM Algorithms with Constraint Engineering Applications. In: Samui, P., Sekhar, S. and Balas, V.E., Eds., Handbook of Neural Computation, Elsevier, Amsterdam, 87-107. https://doi.org/10.1016/B978-0-12-811318-9.00005-3

[108] Maia, A., Ferreira, E., Oliveira, M.C., Menezes, L.F. and Andrade-Campos, A. (2017) 3. Numerical Optimization Strategies for Springback Compensation in Sheet Metal Forming. In: Davim, J.P., Ed., Computational Methods and Production Engineering, Elsevier, Amsterdam, 51-82. https://doi.org/10.1016/B978-0-85709-481-0.00003-3

[109] Lima, C.F., Lobo, F.G., Pelikan, M. and Goldberg, D.E. (2011) Model Accuracy in the Bayesian Optimization Algorithm. Soft Computing, 15, 1351-1371.

https://doi.org/10.1007/s00500-010-0675-y 